\title{
Cost Analysis of Mobility Management Entities of SINEMO
}

\author{
Md. Shohrab Hossain, Mohammed Atiquzzaman \\ School of Computer Science, University of Oklahoma, \\ Norman, OK 73019. \\ Email: \{shohrab, atiq\}@ou.edu
}

\author{
William Ivancic \\ NASA Glenn Research Center \\ Cleveland, OH 44135. \\ Email: wivancic@grc.nasa.gov
}

\begin{abstract}
Seamless IP-diversity based NEtwork MObility (SINEMO) was proposed to address a number of drawbacks of the Network Mobility (NEMO) protocol that manages networks in motion. Increasing number of mobile hosts results in higher level of signalling cost on the mobility agents in a mobility protocol. Previous cost analysis on mobility protocols have not considered all possible costs for mobility management, resulting in incomplete cost estimation. In this paper, we have developed analytical models to estimate costs of mobility management as functions of network size, mobility rate, traffic rate and data volume for all the entities of SINEMO. Numerical results, comparing the cost between mobility entities of SINEMO and NEMO, reveal that SINEMO has lower cost yet higher efficiency than NEMO. Our comprehensive cost model can be used as a framework for estimating total cost of key mobility management entities of different handover protocols, and can aid in decision making to choose the most efficient protocol for future all-IP mobile and wireless networks.
\end{abstract}

Index Terms-Network mobility, mathematical modeling, signaling cost, seamless handover, mobility protocols.

\section{INTRODUCTION}

To facilitate continuous Internet connectivity of hosts moving together, IETF proposed NEtwork MObility Basic Support Protocol (NEMO BSP) [1]. NEMO BSP has a number of limitations: high handover latency, packet loss, and inefficient routing path, giving rise to deployment issues. To address these drawbacks, we earlier proposed SINEMO, an IP diversitybased seamless network-mobility management scheme [2].

In a mobile computing environment, a number of network parameters (such as, network size, mobility rate and traffic rate) influence the costs resulting from mobility protocols. These include costs incurred in updating location manager about the change of location, sending updates to hosts with ongoing communication, and processing and lookup costs by various mobility agents, etc. With the rapid growth and popularity of mobile and wireless networks, increasingly larger number of IP-enabled mobile devices now require support from the mobility management entities (e.g., location manager, mobile router, etc.). The expansion of network size incurs additional load on mobility management entities, resulting in the performance degradation of mobility protocols.

There have been earlier attempts for cost analysis [3]-[6] of mobility protocols. Fu et al. [3] analyze the signaling costs

The research work reported in this paper was supported by NASA Grant NNX06AE44G. of SIGMA and HMIPv6. Reaz et al. [4] perform the signaling cost analysis of SINEMO but does not consider all possible costs of all the entities. Makaya et al. [5] present an analytical model for the performance and cost analysis of IPv6-based mobility protocols. Xie et al. [6] have analyzed various handoff scenarios for a dual stack mobile node roaming in a mixed IPv4/IPv6 environment. However, the above studies [3]-[6] did not consider all possible costs for mobility management e.g., costs related to query messages by $\mathrm{CN}$, refreshing binding updates, registration messages, securing location updates and data acknowledgement messages, etc. Moreover, they did not compute the costs for various mobility management entities of the protocol. Hence, those analyses are incomplete.

The objective of this paper is to perform a comprehensive cost analysis of mobility entities of SINEMO, compare them with that of NEMO BSP. The contributions of this work are: (i) developing analytical models to estimate the total costs of various mobility management entities of SINEMO, (ii) comparing between the total costs and efficiency of SINEMO and NEMO in terms of network size, mobility rate and traffic rate.

Results show that SINEMO has lower cost yet higher efficiency than NEMO irrespective of session lengths, network size and mobility rate since SINEMO uses optimal route in data delivery between the mobile network and an arbitrary node in the Internet. This analytical model can be used as a framework to estimate costs of different handover protocols, and can aid in decision making to choose the most efficient protocol for future all-IP mobile and wireless networks.

The rest of the paper is organized as follows. Section II briefly explains SINEMO architecture. The cost models OF SINEMO entities are presented in Section III, followed by comparative numerical results between SINEMO and NEMO in Section IV. Finally, Section V has the concluding remarks.

\section{SINEMO ARCHITECTURE}

Fig. 1 shows the architecture of SINEMO [2]. The Mobile Network (MN) consists of multi-homed Mobile Routers (MR) which can be connected to two wireless networks exploiting IP-diversity. The MR acts as a gateway between the hosts and the Access Routers (ARs) for Internet access. Correspondent node $(\mathrm{CN})$ sends traffic to a node in the MN termed as Mobile Network Node (MNN) which can either be a Local Fixed Node (LFN), Local Mobile Node (LMN), or Visiting Mobile Node 


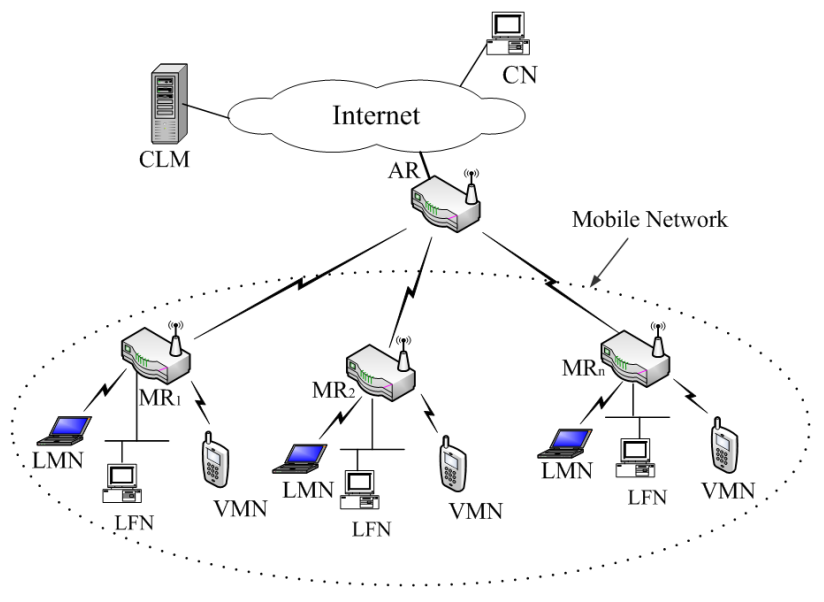

Fig. 1. SINEMO Architecture

(VMN). A Central Location Manager (CLM) maintains the IP addresses of MRs in an MN. A Local Location Manager (LLM), usually co-located with the MR, is used to keep the IP addresses of the hosts inside the MN. When a MN moves into one subnet, MR obtains its own public IP address and one or more address prefixes. MR provides and reserves an IP address for each host which only uses private addresses for connectivity. After handover, only the public addresses are modified at MR, the private IP addresses of the hosts remain unchanged. MR thus hides mobility from the hosts. The readers can refer to [2] for more details of SINEMO handover and location management.

\section{COST ANALYSIS}

In this section, we perform the cost analysis of the key entities of SINEMO: CLM, MR (collocated with LLM) and the complete network.

\section{A. Notations}

The notations used in this paper are listed below.

$N_{r} \quad$ Number of mobile routers in the MN,

$N_{f} \quad$ Number of LFNs in the MN,

$N_{l} \quad$ Number of LMNs in the MN,

$N_{v} \quad$ Number of VMNs in the MN,

$N_{m n n}$ Total MNNs in the MN, i.e., $N_{m n n}=N_{f}+N_{l}+N_{v}$,

$N_{m} \quad$ Number of mobile hosts, i.e., $N_{m}=N_{l}+N_{v}$,

$N_{c} \quad$ Number of CNs communicating with all MNNs,

$\delta_{L} \quad$ Per hop transmission cost for Location Update (LU),

$\delta_{A L} \quad$ Per hop transmission cost for aggregated location update message,

$\delta_{B} \quad$ Per hop transmission cost for Binding Update (BU),

$\delta_{Q} \quad$ Per hop transmission cost for query message,

$\delta_{R} \quad$ Per hop transmission cost for registration message,

$\delta_{D T}$ Per hop transmission cost for each data packet,

$\delta_{D A} \quad$ Per hop transmission cost for each (data) Ack packet,

$\delta_{R R}$ Per hop transmission cost for Return Routability (RR) message,

$\delta_{D H} \quad$ Per hop transmission cost for DHCPv6 message,

$\sigma \quad$ Proportionality constant (for transmission cost) of wireless link over wired link, $\psi \quad$ Linear coefficient for lookup cost,

$T_{r} \quad$ Subnet residence time,

$\lambda_{s} \quad$ Average session arrival rate,

$h_{p} \quad$ Average number of hops between Internet to arbitrary $\mathrm{CN}$ or CLM or AR,

$h_{i n} \quad$ Average number of hops in the Internet,

$\gamma_{l} \quad$ Unit processing cost at LM,

$\kappa \quad$ Maximum transmission unit,

$\alpha \quad$ Average session length (data file size).

\section{B. Assumptions}

Following are the assumptions of the model:

- Session arrival rate for each mobile host is equal.

- The data (file) size in each session is equal.

- Each CN has one ongoing session with a MNN.

- Binary search is used to search location database.

\section{Central Location Manager}

In SINEMO, the CLM keeps the location database of the mobile network and has the tasks of processing LU, BU, RR, and query messages.

1) Location update messages: When MN crosses subnets, MR acquires new IP address from the foreign network and notifies the CLM using LU message. The LU contains the new address of the LLM and the public addresses of the MHs inside the MR's domain. Each of the $N_{r}$ MRs sends such aggregated LU to the CLM which sends back acknowledgement. The CLM has to process the LU message and update the location database of all the nodes inside the MN. Thus the cost (transmission and processing) on the CLM due to the LU messages is given by,

$$
\Gamma_{C L M}^{L U}=\frac{N_{r}\left(\delta_{A L}+\delta_{L}\right)+\left(N_{m}+N_{f}\right) \gamma_{l}}{T_{r}}
$$

2) Query message: At the beginning of every session between CNs and MNNs, query messages are sent to CLM which requires the following transmission cost:

$$
\Gamma_{C L M}^{Q R}=2 N_{c} \delta_{Q} \lambda_{s}
$$

3) Table lookup: When $\mathrm{CN}$ initiates a connection (query) request for a node inside the $\mathrm{MN}$, it requires a lookup at CLM which is proportional to the logarithm of the number of MNNs.

$$
\Gamma_{C L M}^{L P}=N_{c} \psi \lambda_{s} \log _{2}\left(N_{m}+N_{f}\right)
$$

4) Refreshing update messages: During the subnet residence time, each MR sends refreshing BU to the CLM and all the CNs so that the binding entry is not expired. If the lifetime of each binding entry is $T_{e}$, then the frequency of refreshing BUs sent to the CLM is $\eta_{r}\left(=\left\lfloor\frac{T_{r}}{T_{e}}\right\rfloor / T_{r}\right)$. Thus, the cost of CLM is as follows:

$$
\Gamma_{C L M}^{R B U}=\eta_{r} N_{r}\left(\delta_{A L}+\delta_{L}\right)
$$


5) Return routability messages: We assume that SINEMO employs RR test to prevent session hijacking similar to the mechanism employed in route optimization of MIPv6 [7]. This test verifies that the node (sending BU) can actually respond to packets sent to a given CoA. Before each BU message, RR messages are exchanged among the MH, CLM and CN. The CLM receives the Home Test Init (HoTI) message sent by the $\mathrm{MH}$ and forwards it to the CN. CLM also receives the Home Test (HoT) message from the CN and sends it back to MH. This happens for every $T_{r}$ seconds and for every MH-CN pair under the MN. Therefore, cost on CLM for RR messages,

$$
\Gamma_{C L M}^{R R}=N_{c} \frac{4 \delta_{R R}}{T_{r}}
$$

Thus, the total cost of the CLM can be obtained by adding Eqns. (1), (2), (3), (4), and (5):

$$
\Gamma_{C L M}=\Gamma_{C L M}^{L U}+\Gamma_{C L M}^{Q R}+\Gamma_{C L M}^{L P}+\Gamma_{C L M}^{R B U}+\Gamma_{C L M}^{R R}
$$

\section{Mobile Router}

In SINEMO, the MR has the following costs:

1) Acquiring IP address and prefixes: MRs acquire IP addresses and prefixes from the AR in the foreign network during each handoff by exchanging DHCPv6 request-reply messages through the wireless media. Therefore,

$$
\Gamma_{M R}^{D H C P}=\frac{2 \sigma \delta_{D H}}{T_{r}}
$$

2) Updating NAT table: After acquiring the IP addresses for the nodes inside the MN, MR reserves public IP addresses for the MNNs and modifies the NAT table whose size is proportional to $\left(N_{m}+N_{f}\right) / N_{r}=N_{m n n}^{m r}$ (let). Thus, the cost of updating such table is as follows:

$$
\Gamma_{M R}^{N A T}=\frac{\psi\left(N_{m}+N_{f}\right)}{N_{r}} \log _{2}\left(\frac{N_{m}+N_{f}}{N_{r}}\right)
$$

3) Updating sessions table and sending $B U$ to $C N s$ : The MR keeps a table known as Sessions table that records the CN-MNN pair of the ongoing sessions. Each entry of the sessions table is a triple with CN's IP address, MNN's current public address and MNN's private IP-address. After acquiring the IP address and prefixes at each handoff, the MR uses the newly assigned public addresses (to the MNNs in the NAT table) to modify the session table of size proportional to number of sessions. Among the $N_{c}$ sessions, there will be $N_{c} / N_{r}$ sessions through each MR. Thus updating the session table have a cost proportional to $\frac{N_{c}}{N_{r}} \log _{2}\left(\frac{N_{c}}{N_{r}}\right)$. In addition, each MR sends BUs to (and receive binding acknowledgement from) the CNs and the transmission cost associated with these BUs is $2 \sigma \delta_{B}\left(N_{c} / N_{r}\right)$ in every handoff. Therefore,

$$
\Gamma_{M R}^{B U}=\frac{N_{c} \log _{2}\left(N_{c} / N_{r}\right)+2 \sigma \delta_{B}\left(N_{c}\right)}{N_{r} T_{r}}
$$

4) Location updates to CLM: After each handoff, each MR sends LU to CLM informing newly acquired IP address and prefixes which costs the following:

$$
\Gamma_{M R}^{L U}=\frac{\sigma\left(\delta_{A L}+\delta_{L}\right)}{T_{r}}
$$

5) MH's local registration messages: When a MH enters a new MR domain in every $T_{l}$ sec (let), it receives router advertisements, and registers with the MR The MR acts as the LLM, assigns an ID to the MH and updates a local table with public IP address. Therefore,

$$
\Gamma_{M R}^{L R}=\frac{N_{m}}{N_{r}} \times \frac{2 \sigma \delta_{R}+\gamma_{l}}{T_{l}}
$$

6) Refreshing update messages: MR sends refreshing $\mathrm{BU}$ to CLM and the CNs with a frequency of $\eta_{r}$ which costs the following for the MR:

$$
\Gamma_{M R}^{R B U}=\sigma \eta_{r}\left(\delta_{A L}+\delta_{L}\right)\left(1+N_{c} / N_{r}\right)
$$

7) Data delivery cost: In every CN-MNN session, $\left\lceil\frac{\alpha}{\kappa}\right\rceil$ data packets are sent along with corresponding ACK. As each MR manages $N_{c} / N_{r}$ sessions, total data / ACK packet arrival rate to a MR is $\lambda_{p}=\left(N_{c} / N_{r}\right) \lambda_{s}\left\lceil\frac{\alpha}{\kappa}\right\rceil$. Each data packet arriving from $\mathrm{CN}$ is intercepted by MR which modifies the destination address by private IP address searching the NAT table of size proportional to $N_{m n n}^{m r}$. The opposite is done for reverse path. Moreover, transmission cost is incurred through the wireless media. Therefore, data delivery cost at each MR is given by,

$$
\Gamma_{M R}^{D D}=\lambda_{p}\left(\psi \log _{2} N_{m n n}^{m r}+\sigma\left(\delta_{D T}+\delta_{D A}\right)\right)
$$

8) Return routability messages: To prevent session hijacking RR messages are exchanged through MR whose cost is,

$$
\Gamma_{M R}^{R R}=\frac{4 \sigma N_{m n n}^{m r} \delta_{R R}}{T_{r}}
$$

Therefore, the total cost of each MR can be obtained by adding Eqns. (7), (8), (9), (10), (11), (12), (13), and (14):

$$
\begin{aligned}
\Gamma_{M R} & =\Gamma_{M R}^{D H C P}+\Gamma_{M R}^{N A T}+\Gamma_{M R}^{B U}+\Gamma_{M R}^{L U}+\Gamma_{M R}^{L R} \\
& +\Gamma_{M R}^{R B U}+\Gamma_{M R}^{D D}+\Gamma_{M R}^{R R}
\end{aligned}
$$

\section{E. Complete Network}

In order to compute the total cost of the network as a whole, we consider all the resources (such as, bandwidth, processing power etc) consumed in all network entities.

1) Query message: The transmission costs for the queryreply messages between $\mathrm{CN}$ and CLM are $N_{c}\left(2\left(h_{p}+\right.\right.$ $\left.\left.h_{\text {in }}+h_{p}\right) \delta_{Q}\right) \lambda_{s}$ whereas the lookup cost in the CLM is $N_{c} \psi \lambda_{s} \log _{2}\left(N_{m}+N_{f}\right)$. Hence,

$\Gamma_{N e t}^{Q R}=2 \lambda_{s} N_{c}\left(2 h_{p}+h_{i n}\right) \delta_{Q}+\psi \lambda_{s} N_{c} \log _{2}\left(N_{m}+N_{f}\right)$

2) Local registration messages: Every subnet crossing by a MH (in every $T_{l} \mathrm{sec}$ ) within a MR region, triggers a local registration message towards MR. This incurs transmission and processing cost at the MR as follows.

$$
\Gamma_{\text {Net }}^{L R}=N_{m} \frac{2 \sigma \delta_{R}+\gamma_{l}}{T_{l}}
$$

3) Return routability messages: Each HoTI and HoT messages follow a path of $\left(h_{p}+h_{i n}+h_{p}\right)$ wired hops and one wireless hop between MR and CLM whereas the path between CLM and CN contains $\left(h_{p}+h_{i n}+h_{p}\right)$ wired hops. Each CoTI message is sent directly to $\mathrm{CN}$ from the MR which contains $\left(h_{p}+h_{i n}+h_{p}\right)$ wired hops and one wireless hop. Therefore, cost for RR messages are as follows:

$$
\begin{aligned}
\Gamma_{N e t}^{R R} & =\frac{N_{c}}{T_{r}} \times 2 \delta_{R R}\left(\left(h_{p}+h_{i n}+h_{p}+\sigma\right)+\left(h_{p}+h_{i n}+h_{p}\right)\right. \\
& \left.+\left(h_{p}+h_{i n}+h_{p}+\sigma\right)\right)
\end{aligned}
$$


4) Location updates: After each handoff, MRs send LUs to the CLM informing the newly acquired IP address and prefixes. As the CLM is $\left(h_{p}+h_{i n}+h_{p}\right)$ wired hops and one wireless hop away from the MR, the cost of the network for LU message is given by,

$$
\Gamma_{N e t}^{L U}=\frac{N_{r}\left(\delta_{A L}+\delta_{L}\right)\left(h_{p}+h_{i n}+h_{p}+\sigma\right)+N_{r} \gamma_{l}}{T_{r}}
$$

5) Binding updates to CNs: BUs by the MR go through one wireless hop, and $\left(h_{p}+h_{i n}+h_{p}\right)$ wired hops to reach a $\mathrm{CN}$. In addition, processing cost of $N_{c} \log _{2}\left(N_{c} / N_{r}\right)$ are incurred at $N_{r}$ MRs. Thus cost for BUs to CNs are given by,

$$
\Gamma_{N e t}^{B U}=\frac{2 \delta_{B} N_{c}\left(2 h_{p}+h_{i n}+\sigma\right)+N_{c} \log _{2}\left(N_{c} / N_{r}\right)}{T_{r}}
$$

6) Refreshing update messages: Each MR sends $\eta_{r}$ refreshing BUs to CLM and all CNs in every $T_{r}$, thereby incurring the following cost:

$$
\Gamma_{N e t}^{R B U}=\frac{\eta_{r} N_{r}}{T_{r}} \times\left(\sigma\left(\delta_{A L}+\delta_{L}\right)+2 \sigma N_{c} \delta_{B}\right)
$$

7) Data delivery cost: The data and ack packets travel directly through $\left(h_{p}+h_{i n}+h_{p}\right)$ wired and one wireless hops to reach the MR which updates destination address and forward it to MNN. Thus,

$\Gamma_{N e t}^{D D}=\lambda_{p} N_{r}\left(\psi \log _{2} N_{m n n}^{m r}+\left(2 h_{p}+h_{i n}+\sigma\right)\left(\delta_{D T}+\delta_{D A}\right)\right)$

Therefore, total cost on complete network due to SINEMO protocol can be obtained by adding Eqns. (16), (17), (18), (19), (20), (21) and (22):

$$
\Gamma_{N e t}=\Gamma_{\text {Net }}^{Q R}+\Gamma_{\text {Net }}^{L R}+\Gamma_{\text {Net }}^{R R}+\Gamma_{\text {Net }}^{L U}+\Gamma_{\text {Net }}^{B U}+\Gamma_{\text {Net }}^{R B U}+\Gamma_{\text {Net }}^{D D}
$$

\section{F. Efficiency of SINEMO protocol}

We define a performance metric to evaluate the efficacy of mobility protocols in terms of signaling costs since no such metric exists. Efficiency of a mobility protocol is defined as the ratio of data delivery cost (when an optimal route is used) to the total cost (that includes signaling and data delivery costs) required for the mobility protocol. The net data delivery cost of SINEMO can be obtained as follows:

$$
\Gamma^{D D}=\lambda_{p} N_{r}\left(2 h_{p}+h_{i n}+2 \sigma\right) \delta_{D T}
$$

Hence, efficiency of SINEMO protocol can be obtained as:

$$
\zeta^{S}=\frac{\Gamma^{D D}}{\Gamma_{N e t}}
$$

\section{Numerical Results}

In this section, we present numerical results demonstrating the impact of network size, mobility rate, traffic rate and data volume on the total cost of various mobility management entities of SINEMO and NEMO, along with the comparison between their efficiencies. The cost analysis of NEMO protocol entities is presented in [8] and we use it for our comparison in this section. The values for the system parameters are consistent with [4], [8], [9]: $\delta_{L}=0.6, \delta_{A L}=1.4, \delta_{B}=0.6$, $\delta_{Q}=0.6, \delta_{D H}=1.4, \delta_{R R}=0.6, \delta_{D T}=5.72, \delta_{D A}=0.60, \sigma$ $=10, \lambda_{s}=0.01, \gamma_{t}=10, N_{c}=N_{m n n}, h_{i n}=5, h_{p}=1, T_{r}=$ $70 \mathrm{~s}, T_{e}=60 \mathrm{~s}, T_{l}=0.1 T_{r}, \psi=0.3, \alpha=10 \mathrm{~Kb}$, and $\kappa=576 \mathrm{~b}$, $N_{r}=20, N_{f}=70, N_{l}=100, N_{v}=100, N_{m}=200$.

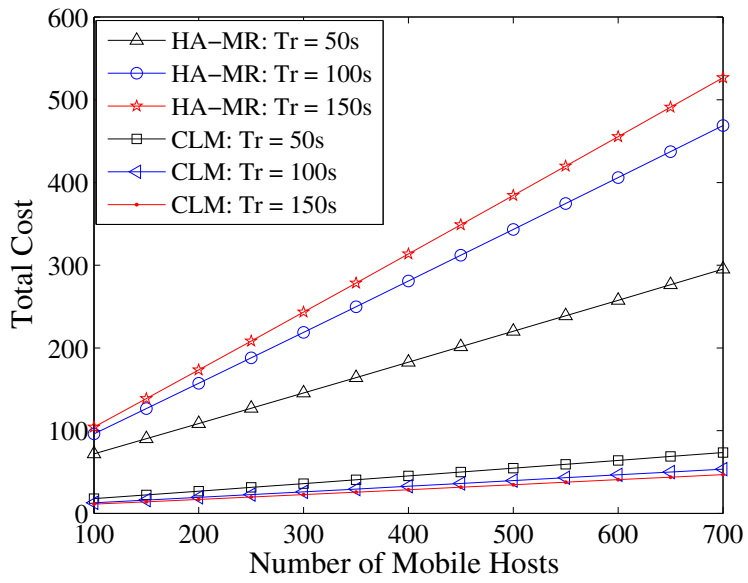

Fig. 2. Impact of number of mobile hosts on the total cost of the HA-MR and CLM for different subnet residence times.

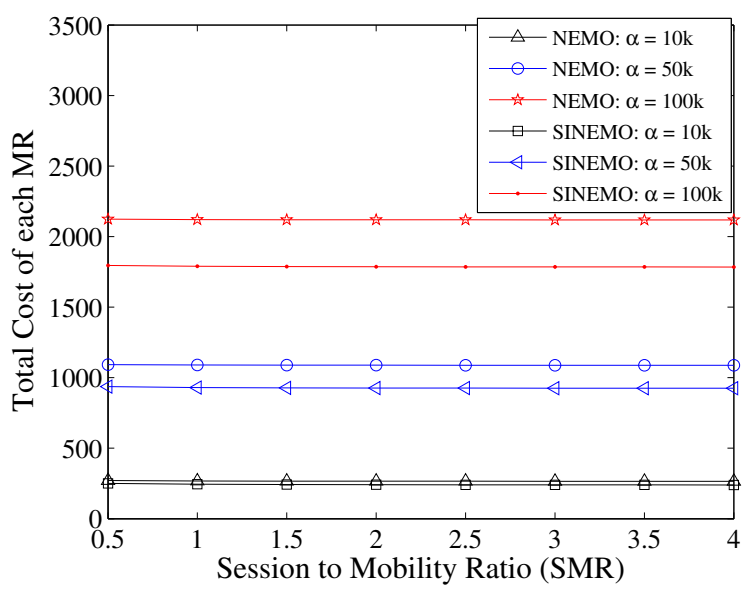

Fig. 3. Impact of SMR on the total cost of each MR for different session lengths.

\section{A. Cost of CLM vs. HA-MR}

We compare NEMO's HA-MR with SINEMO's CLM as their tasks are similar. For Fig. 2, $N_{v}=N_{l}=\frac{1}{2} N_{m}$, with $N_{f}=100$ and $N_{r}=20$. The cost of NEMO's HA-MR is found to be much higher than that of SINEMO's CLM as the first data packets of each session are routed through HA-MR. Total costs of HA-MR and CLM increase for higher number of MHs. However, in terms of $T_{r}$, total cost of CLM and HA-MR behave just opposite. For NEMO, when $T_{r}$ increases, refreshing binding cost increases, although costs related to handoff reduces due to lower handoff frequency. Other costs (query and data delivery) remain unchanged. The net result is increase of total cost. For SINEMO, the effect of refreshing BUs are much less than that related to handoff costs, thereby producing reduced total cost.

\section{B. Cost of each $M R$}

In Fig. 3, the total costs of each MR (for NEMO and SINEMO) are shown as a function of Session to Mobility Ratio (SMR) which is defined as $\lambda_{s} \times T_{r}$. Higher session length causes more data packets to be routed through each $\mathrm{MR}$, resulting in higher cost. The total cost is found to be 


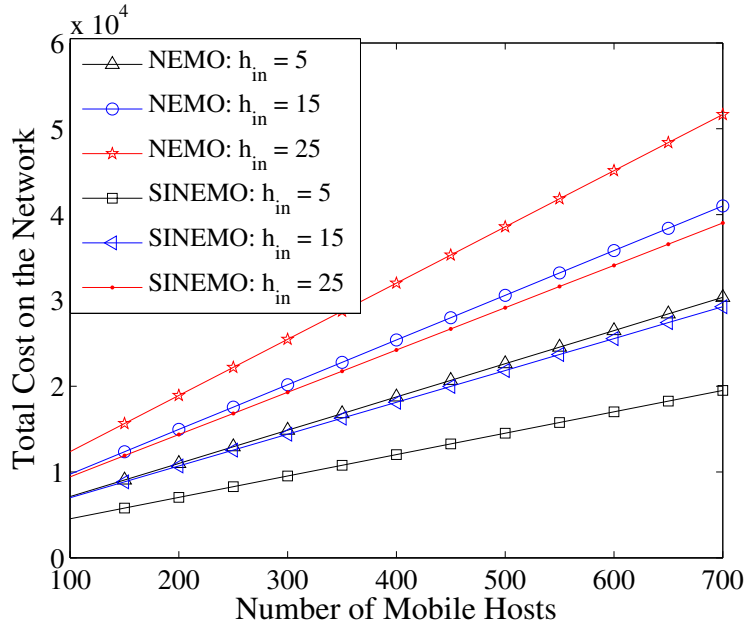

Fig. 4. Impact of number of mobile hosts on the total cost of the network for different number of hops in Internet.

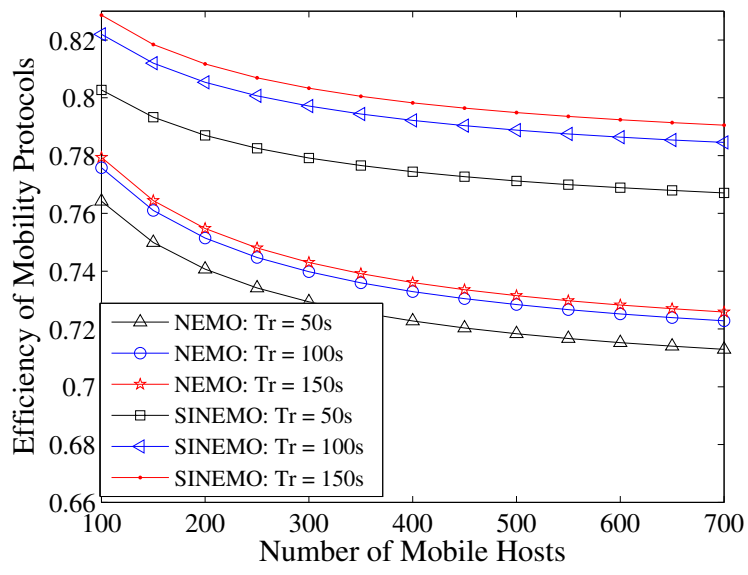

Fig. 5. Efficiency of SINEMO and NEMO vs. number of MHs for various subnet residence times.

invariant of SMR due to the dominance of data delivery cost while NEMO having higher cost than SINEMO in each case.

\section{Complete Network}

The total cost of the complete network is shown as a function of number of mobile hosts in Fig. 4. Increased number of mobile hosts sends higher number of location and binding updates; in addition, query for the mobile hosts also increases for higher number of mobile hosts in the MN. The total cost is shown for different number of hops $\left(h_{i n}\right)$ in the Internet.The slope of the total cost rises for higher values of $h_{i n}$ since its value influences all the costs of the network.

\section{Efficiency of SINEMO vs. NEMO}

In Fig. 5, the efficiency of SINEMO is found to be higher than that of NEMO, as SINEMO uses direct route to send/receive data packets between MNNs and CNs. Efficiency of each protocol increases for higher subnet residence times as the costs related to mobility signaling reduces due to fewer number of handoffs.

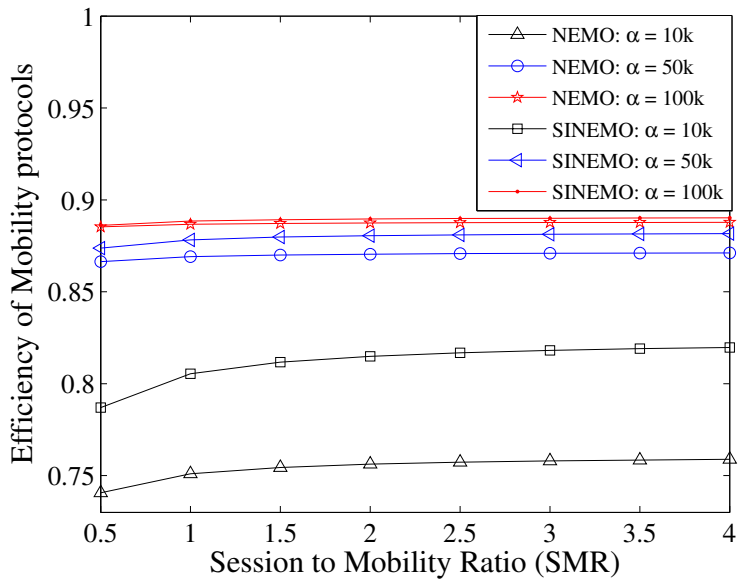

Fig. 6. Efficiency of SINEMO and NEMO BSP vs. SMR for various session lengths.

In Fig. 6, the efficiency of both the protocols increases for increased session lengths since the ratio of signaling traffic to data traffic becomes smaller. However, SINEMO shows a higher efficiency than NEMO irrespective of session lengths.

\section{CONClusion}

In this paper, we have developed analytical models to estimate total costs of mobility management entities of SINEMO and have compared the results with NEMO. Our results show that the SINEMO has lower cost yet higher efficiency than NEMO irrespective of session lengths, network size and mobility rate. Our comprehensive cost model can be used as a framework for estimating total cost of key mobility management entities of other handover protocols, and can aid in decision making to choose the most efficient protocol for future all-IP mobile and wireless networks.

\section{REFERENCES}

[1] V. Devarapalli, R. Wakikawa, A. Petrescu, and P. Thubert, "NEtwork MObility (NEMO) basic support protocol," RFC 3963, Jan 2005.

[2] P. Chowdhury, M. Atiquzzaman, and W. Ivancic, "SINEMO: An IPdiversity based approach for network mobility in space," in Second IEEE International Conference on Space Mission Challenges for Information Technology (SMC-IT), July 17-21, 2006.

[3] S. Fu and M. Atiquzzaman, "Signaling cost and performance of SIGMA: A seamless handover scheme for data networks," Wireless Communication and Mobile Computing, vol. 5, no. 7, pp. 825-845, Nov 2005.

[4] A. S. Reaz, P. K. Chowdhury, and M. Atiquzzaman, "Signaling cost analysis of SINEMO: Seamless End-to-End Network Mobility," in First ACM/IEEE International Workshop on Mobility in the Evolving Internet Architecture, San Francisco, CA, Dec 01, 2006.

[5] C. Makaya and S. Pierre, "An analytical framework for performance evaluation of IPv6-based mobility management protocols," IEEE Transactions on Wireless Communications, vol. 7, no. 3, pp. 972-983, March 2008.

[6] J. Xie and U. Narayanan, "Performance analysis of mobility support in IPv4/IPv6 mixed wireless networks," IEEE Transactions on Vehicular Technology, vol. 59, no. 2, Feb 2010.

[7] D. Johnson, C. E. Perkins, and J. Arkko, "Mobility support in IPv6," IETF RFC 3775, June 2004.

[8] M. S. Hossain and M. Atiquzzaman, "Cost analysis NEMO protocol entities," University of Oklahoma, Technical report, TR-OU-TNRL-10105, September 2010.

[9] P. Chowdhury, A. Reaz, M. Atiquzzaman, and W. Ivancic, "Performance analysis of SINEMO: Seamless IP-diversity based Network Mobility," in ICC 2007, Glasgow, Scotland, June 24-28, 2007. 